\title{
Energy-awareness in Multihop Routing
}

\author{
Antonio Junior \\ SITI, University Lusofona \\ Email: antonio.junior@ulusofona.pt
}

\author{
Rute Sofia \\ SITI, University Lusofona \\ Email: rute.sofia@ulusofona.pt
}

\author{
António Costa \\ ALGORITMI, Minho University \\ Email: costa@di.uminho.pt
}

\begin{abstract}
This paper discusses and validates energy-aware routing metrics which can be applied to any available routing protocol. We validate the metrics performance based on the two main branches of multihop routing, namely, link-state and distance-vector approaches. The validation is performed based upon discrete event simulations.
\end{abstract}

Index Terms-Multihop routing; energy-efficiency; wireless user-centric networks.

\section{INTRODUCTION}

Nowadays, advances in wireless technology such as Wireless Fidelity (Wi-Fi), gave rise to new types of portable devices and to new types of connectivity models, e.g., User-centric Networks (UCN) [1]. Examples of such environments can be a network formed on-the-fly after a disaster of some nature or even a municipality network where there are nodes that are end-user devices sharing the Internet access.

In such environments, energy-efficiency is also becoming a key aspect. The mentioned devices are portable and heterogeneous in terms of battery. They extend the network capillarity and the operator's control is limited in these environments. It is therefore necessary to consider ways to make these devices operate more efficiently, being one of such aspects the routing performed across such networks. Energy efficiency is today a hot topic, and there is a global effort concerning ways to improve the network operation in regards to such efficiency. Moreover, in the context of the Internet Engineering Task Force (IETF), there is a working group dedicated to the topic of energy efficiency in low power and lossy networks - UCNs are a potential example of such environments.

On the other hand, the environments mentioned rely on multihop routing approaches which are based on shortest-path computation. Routing and the path selection has impact on energy efficiency. Hence, when performing path computation, current routing protocols could consider ways to become more energy aware, more efficient, and robustness is improved.

This line of thought has been debated and validated in our previous work [2], [3], where we have proposed and validated a new set of routing metrics which provide nodes with an energy-awareness ranking based on existing notions such as energy consumption models, energy capacity of a node, as well as residual energy of a node. Out of such work stem two types of metrics: i) a metric that takes into consideration the perspective of a single node (father), the Energy-awareness Node Ranking (ENR); ii) a second metric that takes into consideration the perspective of both the father and son nodes, the Energy-awareness Father-Son (EFS) metric. Such metrics have been validated in the context of the Ad-Hoc on-demand Distance Vector (AODV) protocol [4] and we have proved significant improvements when applied to this protocol.

This paper is a follow-up of such work, being the motivation to analyze whether or not our proposed metrics are beneficial independently of the routing protocol, in multihop environments. Therefore, to validate such aspects, we now compare the metrics both in the context of AODV and of the Optimized Link State Routing (OLSR) protocol [5], based on discrete event simulations and also based on real data set traces.

To evaluate the metrics, we consider the notion of network lifetime. We follow the definition where network lifetime is associated to the time period since a topology becomes active, until the topology becomes disconnected, from a destination reachability perspective.

The rest of this paper is organized as follows. Section II describes related work focused on multi-hop energy-efficiency while section III discusses the energy-awareness in UCNs. Section IV describes our proposed metrics focusing on energyawareness impact on multihop routing approaches. Then, in section $\mathrm{V}$, we present the evaluation settings and section VI show the results focusing on improve energy efficiency. Conclusions and next steps are presented in section VII.

\section{RELATED WORK}

Relevant proposals [6], [7], [8] making multihop routing adaptive, have explored new metrics with different types of optimization, e.g., reduction of energy spent across a path, considering the residual energy capacity of a node or avoiding nodes with low residual energy, on the global network. C. K. Toh [9] discusses different routing properties to consider in multi-hop routing addressing the performance of power efficiency in ad-hoc mobile networks by analyzing four approaches which have as common goal to select an optimal path, being the optimum the minimization of the total power required on the network and also the maximization of the lifetime of all nodes in the network.

The $E^{2} R$ routing protocol [10] uses an opportunistic forwarding scheme to deliver control messages and data packets in a multihop wireless network to energy efficiency in multihop green wireless networks. Unlike other opportunistic routing protocols, it neither uses pre-selected static paths nor prepares forwarding candidates. Previews energy-efficient routing consider a schemes to archive the energy efficiency without specify an energy-aware metric to apply any multi-hop routing protocol. Unless, $E^{2} R$ claim that any routing metric 
can be apply for their routing protocol which a energy-aware is one of them. Some steps towards the definition of energy efficiency metrics for wireless network is outlined by [11]. The approach is to estimate the optimal message size in terms of power consumption, to estimate the average amount of energy spent to transmit one bit and the relationship between traffic and power consumption. However, a energy-aware metric for multihop routing is not defined.

We highlight that the IETF Working Group (WG) Routing Over Low Power and Lossy Networks (ROLL) is currently discussing multihop metrics tailored for energy-efficiency for routing protocols. The WG recently proposed as standard the RFC 6551 [12] which describes a set of link and node routing metrics and constraints, from which the node energy issue is one of them, being suitable for improving routing protocols for Low Power and Lossy Networks (LLNs).

\section{Energy-Awareness in User Centric Networks}

UCNs integrate the end-user connected to the Internet by means of a variety of broadband access technologies, which the final segment is provided by a number of short-range technologies, among which Wi-Fi is a solution [1].

We provide an example of a generic scenario in Figure 1 , where groups of mobile nodes are depicted by a dotted line. Within each group, nodes may move in an independent way according to human movement behavior (social mobility). Furthermore, nodes may also move in groups, also mimicking human social behavior. Groups have a spatial-temporal correlation, e.g., a group at an instant in time may dissolve in a different instant in time and space. The illustrated nodes can be either static or mobile. In addition, nodes may behave as a regular node, or a micro-provider node. A micro-provider node is basically a node that provides Internet access to other nodes. It should be noticed that in contrast to the notion of gateway in MANETs, a micro-provider may simply relay Internet access from a gateway to a group of nodes. In addition, a micro-provider node may be completely mobile. Therefore, the topology presented in Figure 1 shows a highly dynamic behavior, where not only links are bound to frequent changes, but also where the nodes that provide Internet access can also change on-the-fly, e.g., due to congestion of the microprovider(s) in the group, due to better network conditions.

In this scenario, the end-user (or a community of end-users) is a micro-provider in the sense that he/she shares his/her subscribed broadband Internet access based on some incentive scheme. The way people interact and move is a behavior that we can root on social network theory, due to the fact that humans are not only carriers but also the decision makers for the operation of nodes that form the topology. Adding to the variability due to node movement, for instance, another key aspect is that some devices are multimedia capable with strong limitations in terms of energy capabilities.

Hence, energy efficiency is a key aspect since those mobile devices Wi-Fi enabled are heterogeneous in terms of battery capacity and energy consumption. By devising routing metrics to assist multihop routing protocols in becoming more

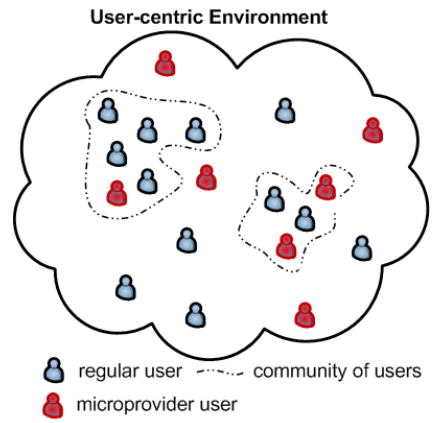

Fig. 1: Generic example of User-Centric Environment.

efficient without adding too much operational complexity, one is improving the overall notion of energy aware networks.

Multihop protocols choosing paths based on optimal network lifetime is an aspect that introduces more flexibility in multihop routing approaches and makes them better suited for user-centric environments. Hence, the idea behind our work is consider energy-aware routing metrics that can provide stability in terms of network lifetime to shortest-path based routing improving the overall network lifetime without penalizing the network performance. The impact of such inclusion of energy-aware metrics should be some minor changes in the control messages of the protocols which can be validate under experimental evaluation.

We emphasize that the architecture and also the energyawareness requirements of wireless sensor networks are different than the ones in user-centric environments. Sensor nodes are normally considered to be homogeneous in terms of energy capability and normally placed to get information and send it to a manager sensor node. In contrast, in user-centric networks, nodes are expected to be heterogeneous in terms of energy and to follow a human behavior as social networking. Those aspects do not allow the energy-efficiency and energyawareness solutions of sensor networks to be reusable in usercentric environments.

\section{BACKGROUND}

In this section we describe our proposed metrics focusing on the impact in current multihop routing approaches.

\section{A. Our Proposed Energy-awareness Metrics}

In previews work [2] we have proposed two sets of metrics, as explained in section I. We also have discussed the energy awareness multihop routing from a single node vs. the two node association perspective. This section provides a overview of the metrics previously proposed, to assist the reader in understanding the line of thought we have adopted, and also to better understand the results extracted in the validation.

The first metric, ENR, provides a single node perspective concerning energy consumption. Such metric, provided by equation 1 , gives a cost to each node $i$ by taking into consideration the time a node spends in idle mode, in comparison to the full operation time of the node. In other words, nodes that have been in idle mode for a long period of time in the 
past and that still have a reasonable large estimated lifetime are better candidates to be elements in a shortest-path.

Hence, we consider the total period in idle time, $t_{\text {idle }}$ over the full lifetime expected for a specific node, which is given by the sum of the elapsed time period $T$ with the estimated lifetime of the node, as provided in equation 1 . The estimated lifetime $C(i)$ provided by [13] have considered the ratio between residual energy and drain rate which can capture the heterogeneous energy capability of nodes.

$$
\operatorname{ENR}(i)=\frac{T-t_{i d l e}}{T \times C(i)}
$$

The smaller $\operatorname{ENR}(i)$ is, the best ranking a node has, which implies that such node has a higher probability of becoming a next hop in a path to a destination.

Following such line of thought, we have also considered whether or not providing a combined perspective of energy consumption between father and son could improve path selection. The reasoning behind this relates to the fact that by combining such perspectives, we can assist a protocol in reacting quicker to energy changes.

The EFS metric, provided by equation 2, is therefore, a sucessor-based perspective which consider a cost of association between two successive nodes (father and son).

$$
\operatorname{EFS}(i, j)=\operatorname{ENR}(i) \times \operatorname{ENR}(j)
$$

The smaller $\operatorname{ESR}(i, j)$ is, the higher the probability that link $i$ and $j$ becomes a segment in the path to the destination.

\section{B. Multihop Routing Approaches, Energy-awareness Impact}

The most popular multihop routing protocols stem from two distinct routing families: link-state, and distance-vector routing. Out of such families, the most popular protocols in use today are AODV (actually working progress AODVv2 [14]) and OLSR (actually working progress OLSRv2 [15]).

In this section we are going to explain main differences concerning these two protocols, as the intent is to assess whether or not we can truly provide metrics that can be applied to any multihop routing approach - the ones mentioned, and others arising now, or in the future.

The purpose of any multihop routing protocol is to dynamically find paths to reach a destination and to select the best path, where best is connoted to the notion of shortest path.

In link state routing the nodes diffuse (depth-first search) a set of their link-state information throughout the whole network. Such packets would contain the identity of the source node, its neighbors, and the cost of routing to them. After the initial network discovery process, all nodes converge to have a perspective of the global topology, at the expense of a higher signaling. Distance vector routing is based on BellmanFord shortest path search algorithm. Nodes therefore keep status concerning destination cost, and next hop towards a destination. Every node periodically broadcasts and kept status concerning found destinations to neighbors.

Operationally, as explained, these two families of protocols have a very different behavior, and applying global metrics to them independently of the protocol behavior is not trivial. However, from an energy-aware perspective, it is possible to do so, by considering that both families rely on shortest-path computation.

Hence, the line of thought considered in the development of our energy-aware metrics is that the principle of shortest-path computation must be kept. Instead of hop-count, a metric that can provide an energy expenditure cost to a node is considered. The main caveat related with this change is that in order to keep accuracy, one must ensure that the protocol synchronizes path status adequately. This implies considering either a timewindow mechanism, or updates to a node's cost each time a change occurs. These are regular techniques, where it is essential to find an adequate commitment between accuracy and low overhead due to the required signaling.

1) Operational Aspects, Distance-Vector: To analyze the behavior of the proposed metrics in distance-vector approaches we have considered AODV. This section describes the operational procedures we have considered so far, to ensure that the protocol can cope with the new metrics.

AODV is distance-vector protocol that works on-demand determining a route to a destination only when a node wants to send a packet to that destination. Routes are maintained as long as there is active traffic to the specific destination. Sequence numbers ensure the freshness of routes and are a way to mitigate transient loops and of avoiding counting-to-infinity. AODV nodes use four types of messages to communicate among each other. In the route discovery process are used Route Request (RREQ) and Route Reply (RREP) messages. Moreover, Route Error (RERR) messages are used for route maintenance; HELLOs are used to keep status concerning links (between neighbors).

The AODV protocol uses a hop count as metric to determine the shortest path between source and destination nodes. The routing information is only exchanged between directly connected neighbors. In order to accommodate our metrics, we have used the reserved field of the control messages to include the energy-aware cost and then use it to determine the shortest path instead of hop count as in native approach. For the node-based perspective, i.e., ENR metric, the protocol uses the node cost to compute the path. For the successorbased perspective, i.e., EFS metric, we have used the HELLO control messages to exchange the node cost and then define a binding cost between two nodes to compute the shortest path. The node cost sent from a node to its neighbors which its neighbors will have this information in advance to improve robustness.

2) Operational Aspects, Link-State: For the case of linkstate we have considered OLSR. As a proactive link-state protocol, OLSR relies on flooding techniques to assist in a quick synchronization of the global topology perspective. OLSR is, however, optimized to the wireless media by relying on HELLO exchange which is capable of performing a 2hop neighbor information discovery and then performing a distributed election of a set of Multipoint Relay (MPR) nodes. MPRs assist in decreasing control traffic overhead, since only 
those nodes are allowed to broadcast topology changes. Nodes use the topology information to compute next hop paths regarding to all nodes in the network by using shortest path hop count metric. We also have used the reserved field of the control messages to accommodate our metrics allowing a node to send to its neighbors its energy level information. Based on that information, each node can have the perception of the energy level to the link towards the neighbors nodes.

In order to allow OLSR to cope with the new metrics, we had to change the MPR selection mechanism. Instead of considering a selection based on shortest-path with a hopcount metric, we consider a selection based on our metrics. For that, when there are more than one 1-hop neighbors covering the same number of uncovered 2-hop neighbors, the one with the better energy cost to the current node is selected as MPR.

Hence, we emphasize that we made minor changes as possible to accommodate our metric in the two main routing families focused on improve energy efficiency without significant operational changes.

\section{Evaluation Settings}

This section provides the evaluation settigns for the ENR and EFS metrics considering the link state and distance vector multihop routing approaches on ns-2.34 simulations.

We have considered the UM-OLSR implementation for ns-2 provided by [16] and default AODV modules. These modules have been changed to reflect the required changes detailed in next section, being the code publicly available here [17].

\section{A. Implementation Aspects}

As explained in section IV, we have used the reserved field of the control messages to include the energy-aware cost and then use it to determine the shortest path instead of hop count as in native approach (here referenced as $A O D V$-native). For the node-based perspective, i.e., ENR metric, the protocol uses the node cost to compute the path (here referenced as AODVENR). For the successor-based perspective, i.e., EFS metric, we have used the HELLO control messages to exchange the node cost and then define a binding cost between two nodes to compute the shortest path. The node cost sent from a node to its neighbors which its neighbors will have this information in advance improve robustness (here referenced as AODV-EFS).

For OLSR (here referenced as OLSR-native), we also have used the reserved field of the control messages to accommodate our energy-aware metrics allowing a node to send to its neighbors its energy level information. Based on that information, each node can have the perception of the energy level to the link towards the neighbors nodes. In order to better running our metrics computing shortest path, we had changed the MPR mechanism to allow to select an MPR node using the energy-aware instead of hop count as defined in native approach. For that, when there are more than one 1hop neighbors covering the same number of uncovered 2-hop neighbors, the one with the better energy cost to the current node is selected as MPR (here referenced as OLSR-ENR and OLSR-EFS).
TABLE I: Scenario parameters.

\begin{tabular}{|c|c|c|}
\hline Scenario & I & II \\
\hline \hline Area & $600 \mathrm{~m} \mathrm{x} \mathrm{600m}$ & $2587 \mathrm{~m} \mathrm{x} \mathrm{2347m}$ \\
\hline Number of nodes & 25 & 20 \\
\hline Movement & static & human behavior \\
\hline Node speed & - & $1 \mathrm{~m} / \mathrm{s}$ \\
\hline Simulation time & $1000 \mathrm{sec}$ & $24 \mathrm{~h}$ \\
\hline Energy parameters & heterogeneous & heterogeneous \\
\hline Traffic model & Poisson (VBR) & Poisson (VBR) \\
\hline Average packet size & 512 bytes & 512 bytes \\
\hline Sending rate & 128 Kbps & 128 Kbps \\
\hline Number of flows & 4,8 and 12 & 4,8 and 12 \\
\hline
\end{tabular}

\section{B. Experimental Environment}

We have considered the ns-2.34 default physical layer parameters with TwoRayGround propagation model. The scenarios are Wi-Fi based with 802.11g parameters.

We have then considered two scenarios. The first scenario is a controlled random topology, where we consider the parameters described in Table I. The second scenario considered is derived from real data traces collected in North Carolina State University (NCSU) [18], where the parameters have been set as provided in Table I, from the traces.

\section{Performance Results}

The results extracted intend to analyze benefits in terms of network lifetime as defined in section I. We also want to understand the impact of the metrics in the overall network performance considering three additional aspects: i) average end-to-end delay, the time a packet takes between source and destination computed per destination and then averaged across all destinations; ii) throughput, the average number of bytes reaching destination nodes, computed first per destination and then averaged across all destinations measured in Kbps; iii) average packet loss, the percentage of packets that does not reach the destination for any reason.

To generate statistical sound results we relied on Akaroa2 tool. All results have been computed within a $95 \%$ confidence interval.

\section{A. Network Lifetime}

To better represent the wireless heterogeneous user-centric environment across different scenarios, based on the parameters described, all of the nodes of the described topologies have been set with initial energy levels picked up randomly but considering that circa of $30 \%$ of nodes will for sure go down before the simulation ends. We have also randomly picked up on and off periods of nodes, but ensuring that circa $10 \%$ $20 \%$ of nodes go down and then come up after $15 \%-25 \%$ of simulation time. The line of thought considered was to create a more real scenario, where there is more variability in terms of energy levels, energy consumption (due to traffic fluctuation), and also due to path availability.

Figure 2 show the average network lifetime for the different approaches regarding scenario I and II. Concerning scenario I (Figure 2a), we show results for the metrics applied to both AODV and OLSR. Consistently, and independently of 


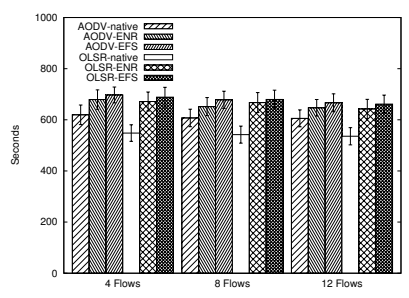

(a) Scenario I.

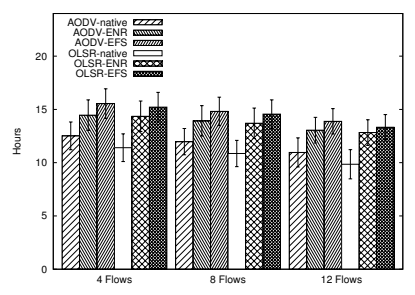

(b) Scenario II.

Fig. 2: Network Lifetime.

TABLE II: Network lifetime improvement.

\begin{tabular}{|c|c|c|c|c|c|c|}
\hline Scenario & \multicolumn{3}{|c|}{ I } & \multicolumn{3}{|c|}{ II } \\
\hline Flows & 4 & 8 & 12 & 4 & 8 & 12 \\
\hline \hline OLSR-ENR & $22.5 \%$ & $23.1 \%$ & $19.9 \%$ & $25.8 \%$ & $26.2 \%$ & $30.2 \%$ \\
\hline OLSR-EFS & $25.6 \%$ & $25.4 \%$ & $23.5 \%$ & $33.4 \%$ & $34.1 \%$ & $35.3 \%$ \\
\hline AODV-ENR & $9.6 \%$ & $7.4 \%$ & $6.8 \%$ & $15.5 \%$ & $16.3 \%$ & $19.1 \%$ \\
\hline AODV-EFS & $12.5 \%$ & $11.7 \%$ & $10.1 \%$ & $24.2 \%$ & $23.7 \%$ & $26.6 \%$ \\
\hline
\end{tabular}

the number of flows, both ENR and EFS result in a better performance than the native protocols, in what concerns network lifetime. When looking at Scenario II (Figure 2b), despite the change of network lifetime unit (seconds to hours), the results still hold, thus corroborating that the metrics result in significant benefits in what concerns network lifetime. To better understand the magnitude of the improvement, Table II shows the relative improvement of network lifetime. When considering OLSR, and in particular OLSR-EFS, the improvement is in the order of $24 \%$ in scenario I, and circa $33 \%$ in scenario II. Moreover, while in scenario I, the improvement seems to lower with the increase in the number of flows, for scenario II, the improvement is higher for a larger number of flows. We believe this is due to the robust path selected by the metrics ranking.

Results achieved with AODV are still high in terms of relative improvement, but lower than the results achieved with OLSR. This is a natural consequence of the way the protocol updates node status, and we believe better results can be achieved, by tackling a better operational design of an extension of AODV. This is something we are considering in future work, as the intent of the current research was to develop energy-aware metrics independently of the underlying protocol. Results achieved with AODV are better for scenario II. We believe this is a consequence of two main aspects: i) the longer periods of simulation due to the traces (where the duration corresponds to days); ii) paths are more stable for longer periods of times, as nodes do not move frequently (or do not appear/disappear as frequently as in scenario I). We make an observation that while scenario I is a controlled topology, scenario II is based on traces, and hences results extracted in this scenario are more relevant to be considered.

\section{B. End-to-end Delay}

As our main goal is to extend network lifetime without penalizing the network operation, Figure $3 \mathrm{a}$ and Figure $3 \mathrm{~b}$

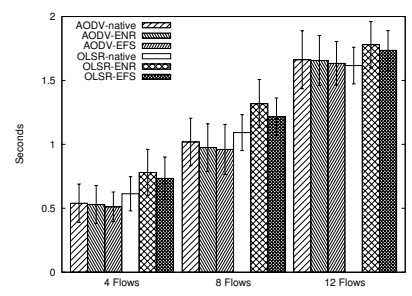

(a) Scenario I.

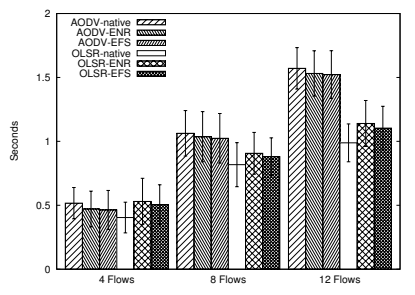

(b) Scenario II.
Fig. 3: End-to-end delay.

TABLE III: End-to-end Delay Improvement.

\begin{tabular}{|c|c|c|c|c|c|c|}
\hline Scenario & \multicolumn{3}{|c|}{$\mathrm{I}$} & \multicolumn{3}{|c|}{ II } \\
\hline Flows & 4 & 8 & 12 & 4 & 8 & 12 \\
\hline \hline OLSR-ENR & $-23.1 \%$ & $-17.7 \%$ & $-7.1 \%$ & $-25.1 \%$ & $-10.1 \%$ & $-14.7 \%$ \\
\hline OLSR-EFS & $-15.6 \%$ & $-8.8 \%$ & $-5.3 \%$ & $-19.9 \%$ & $-7.3 \%$ & $-10.3 \%$ \\
\hline AODV-ENR & $1.9 \%$ & $4.3 \%$ & $0.4 \%$ & $8.9 \%$ & $2.5 \%$ & $2.6 \%$ \\
\hline AODV-EFS & $5.2 \%$ & $5.8 \%$ & $1.7 \%$ & $10.2 \%$ & $3.7 \%$ & $3.2 \%$ \\
\hline
\end{tabular}

show the average end-to-end delay of the ENR and EFS metrics for the different approaches.

In scenario I, the end-to-end delay, even though low across all scenarios experimented, increases (as expected) for higher traffic load. Moreover, while for AODV, ENR and EFS result consistently in a lower end-to-end delay, with OLSR, the metrics achieve a slightly higher end-to-end delay. OLSR-EFS is again the approach that still obtains the best results.

We believe that the reason for OLSR-native to obtain better results is due to our metrics resulting in the selection of a slightly longer shortest-paths, from a hop count perspective. However, the difference (as provided in Table III) is insignificant, and we believe that an OLSR extension can easily take care of this issue, without adding too much operational complexity to the protocol native behavior.

\section{Throughput}

Figure $4 \mathrm{a}$ and Figure 4b show throughput results (Kbps) for different number of flows, again for scenario I and II. The first observation is that when the number of flows increases, the load in the network seems to decrease. This is due to the higher packet loss (refer to section VI-D for results on packet loss) - congested network.

In what concerns AODV results, ENR and EFS slightly

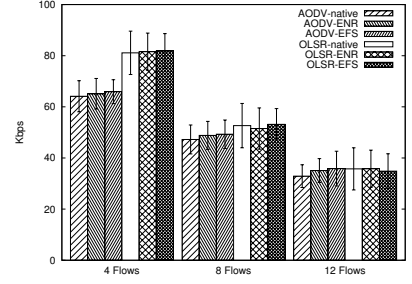

(a) Scenario I.

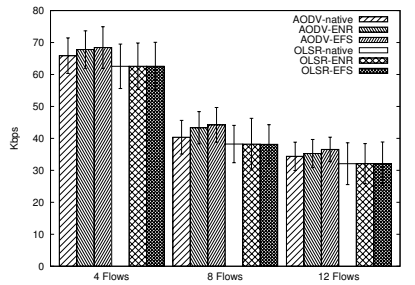

(b) Scenario II.
Fig. 4: Throughput Results. 
TABLE IV: Throughput Improvement.

\begin{tabular}{|c|c|c|c|c|c|c|}
\hline Scenario & \multicolumn{3}{|c|}{$\mathrm{I}$} & \multicolumn{3}{|c|}{ II } \\
\hline Flows & 4 & 8 & 12 & 4 & 8 & 12 \\
\hline \hline OLSR-ENR & $0.5 \%$ & $-2.1 \%$ & $0.1 \%$ & $0 \%$ & $-0.1 \%$ & $0 \%$ \\
\hline OLSR-EFS & $0.9 \%$ & $0.9 \%$ & $-2.5 \%$ & $0 \%$ & $-0.2 \%$ & $0 \%$ \\
\hline AODV-ENR & $1.5 \%$ & $3.4 \%$ & $6.6 \%$ & $2.9 \%$ & $7.4 \%$ & $2.6 \%$ \\
\hline AODV-EFS & $2.8 \%$ & $4.3 \%$ & $8.9 \%$ & $3.8 \%$ & $9.6 \%$ & $6.3 \%$ \\
\hline
\end{tabular}

TABLE V: Packet Loss Improvement.

\begin{tabular}{|c|c|c|c|c|c|c|}
\hline Scenario & \multicolumn{3}{|c|}{ I } & \multicolumn{3}{|c|}{ II } \\
\hline Flows & 4 & 8 & 12 & 4 & 8 & 12 \\
\hline \hline OLSR-ENR & $0.7 \%$ & $-1.5 \%$ & $0 \%$ & $-1.3 \%$ & $0 \%$ & $0.1 \%$ \\
\hline OLSR-EFS & $1.5 \%$ & $-1.1 \%$ & $-0.7 \%$ & $-1.1 \%$ & $-0.2 \%$ & $0.4 \%$ \\
\hline AODV-ENR & $2.4 \%$ & $6.8 \%$ & $6.1 \%$ & $12.3 \%$ & $3.3 \%$ & $1.5 \%$ \\
\hline AODV-EFS & $14.3 \%$ & $9.6 \%$ & $8.1 \%$ & $13.8 \%$ & $3.8 \%$ & $3.9 \%$ \\
\hline
\end{tabular}

improve throughput across all scenarios in comparison to $A O D V$-native which we believe to be due to our metrics resulting in alternative paths (larger-than-shortest-path), whiles AODV always picks the shortest path from a hop count perspective. For OLSR, when the number of flows increases, the metrics and in particular OLSR-EFS, merely match the OLSR-native performance. As mentioned, the intention of the metrics were to provide a more robust path selection in order to improve network lifetime, without jeopardizing the overall network operation. These results show that the metrics are at least as stable as the native versions of the protocols tested, and for AODV, they in fact indirectly improve the network operation. Table IV provides the relative difference in terms of throughput, for a better analysis of the results.

\section{Packet Loss}

Table $\mathrm{V}$ provide results of the relative difference concerning packet loss. The results show our AODV-ENR and AODV-EFS ranking metrics have less average packet loss than $A O D V$ native, which means our metrics selects robust paths in a scenario with more available paths. Considering the OLSR, our metrics is stable comparing to native approach where we believe that there is no impact on overall performance since our main goal is energy efficiency.

\section{CONCLUSions AND NeXt StePs}

In this paper we discuss and evaluate two energy-aware routing metrics, being the intent to show that such metrics can improve multihop routing globally and independently of the protocol flavor. For such purpose, we have considered the two main popular families of multihop routing: linkstate, and distance-vector. In terms of experimentation (based on simulations), we have considered the two most popular examples of each family, respectively: OLSR and AODV. The evaluation that has been carried out show that the metrics significantly improve network lifetime, without incurring significant penalties in terms of network operation. Moreover, and concerning the two proposed metrics, one consistently achieved better results: EFS. We believe that because EFS is based on the ranking (energy-awareness cost) of both a father node and its successor(s), it assists protocols in more quickly selecting stable paths. In other words, our belief for this gain relates to the fact that EFS allows nodes to react quicker to energy changes on a path, which will be more robust earlier in time, assuming that nodes have several successors available.

As future work, we are currently improving the specifications concerning the operational procedures required to assist the protocols in performing better. We are also analyzing the application of these metrics to the Routing Protocol for Low-Power and Lossy Networks (RPL) [19], currently being discussed in the IETF ROLL working group.

\section{ACKNOWLEDGMENT}

This work is supported by Fundação Ciência e Tecnologia (FCT) $\mathrm{PhD}$ scholarship number SFRH/BD/44005/2008 and sponsored by national fundings via FCT, in the context of the UCR project PTDC/EEA-TEL/103637/2008.

\section{REFERENCES}

[1] “ULOOP:User-centric Wireless Local-Loop.” EU IST FP7 Project.

[2] A. Junior, R. Sofia, and A. Costa, "Energy-awareness metrics for multihop wireless user-centric routing," in The 2012 International Conference on Wireless Networks (ICWN'12), July 2012.

[3] A. Junior, R. Sofia, and A. Costa, "Energy-efficient heuristics for multihop routing in user-centric environments," in 12th International Conf. on Next Generation Wired/Wireless Networking (NEW2AN), August 2012.

[4] C. E. Perkins, E. M. Belding-Royer, and S. R. Das, "Ad hoc on-demand distance vector (aodv) routing," RFC 3561, IETF, July 2003.

[5] T. Clausen and P. Jacquet, "Optimized Link State Routing Protocol (OLSR),” RFC 3626, IETF, October 2003.

[6] K. Scott and N. Bambos, "Routing and channel assignment for low power transmission in pcs," in 5th IEEE International Conference on Universal Personal Communications, vol. 2, pp. 498-502, Oct 1996.

[7] J.-H. Chang and L. Tassiulas, "Energy conserving routing in wireless ad-hoc networks," in Proceedings of IEEE INFOCOM, pp. 22-31, 2000.

[8] Q. Xie, C.-T. Lea, M. Golin, and R. Fleischer, "Maximum residual energy routing with reverse energy cost," in Global Telecommunications Conference, IEEE GLOBECOM '03., vol. 1, pp. 564-569, Dec. 2003.

[9] C.-K. Toh, "Maximum battery life routing to support ubiquitous mobile computing in wireless ad hoc networks," IEEE Communications Magazine,, vol. 39, pp. 138-147, June 2001.

[10] T. Zhu and D. Towsley, "E2R: Energy efficient routing for multi-hop green wireless networks," in INFOCOM (WKSHPS), April 2011.

[11] K. Gomez, R. Riggio, T. Rasheed, and F. Granelli, "Analysing the energy consumption behaviour of wifi networks," in IEEE Online Conference on Green Communications (GreenCom), September 2011.

[12] J. Vasseur, M. Kim, K. Pister, N. Dejean, and D. Barthel, "Routing Metrics Used for Path Calculation in Low-Power and Lossy Networks," RFC 6551, IETF, March 2012.

[13] D. Kim, J. J. Garcia-Luna-Aceves, K. Obraczka, J.-C. Cano, and P. Manzoni, "Routing mechanisms for mobile ad hoc networks based on the energy drain rate," IEEE Transactions on Mobile Computing, vol. 2, no. 2, pp. 161-173, 2003.

[14] C. E. Perkins and I. D. Chakeres, "Dynamic MANET On-demand (AODVv2) Routing." draft-ietf-manet-dymo-22 (working in progress), Mar. 2012.

[15] T. Clausen, C. Dearlove, P. Jacquet, and U. Herberg, "The Optimized Link State Routing Protocol version 2." draft-ietf-manet-olsrv2-15 (working in progress), May 2012.

[16] F. J. Ros, "Um-olsr." http://masimum.dif.um.es/?Software:UM-OLSR.

[17] A. Junior, R. Sofia, and A. Costa, "Implementation of energy-aware metrics for aodv and olsr protocols in ns-2." SITI-SW-11-05, May 2011. http://siti.ulusofona.pt/aigaion/index.php/publications/show/126.

[18] I. Rhee, M. Shin, S. Hong, K. Lee, S. Kim, and S. Chong, "CRAWDAD data set ncsu/mobilitymodels (v. 2009-07-23)." Downloaded from http://crawdad.cs.dartmouth.edu/ncsu/mobilitymodels, July 2009.

[19] T.Winter, P. Thubert, A. Brandt, J. Hui, R. Kelsey, P. Levis, K. Pister, R. Struik, J. Vasseur, and R. Alexander, "RPL: IPv6 Routing Protocol for Low-Power and Lossy Networks," RFC 6550, IETF, March 2012. 